\title{
Mucormicosis rinosinusal en un paciente infectado por VIH
}

\author{
LUCÍA AGUAD R., M. DE LA LUZ QUEZADA B., MARITZA RAHAL E., \\ M. PÍA VALLEJOS U., MARIANO MORENO B., JORGE CASTILLO A. y RAFAEL ARAYA V.
}

\section{Rhinosinusal mucormicosis in an HIV positive patient}

Mucormicosis is a fungal opportunistic infection produced by various species of Mucorales, such as Rhizopus, Mucor, Cunninghamella and Absidia. It is associated with diabetes, renal failure, blood diseases, tumors and solid organ or hematological precursors transplants. It presents with diverse clinical manifestations: rhinocerebral, renal, pulmonary, cutaneous, gastrointestinal and disseminated disease. The infection is uncommon in HIV positive patients. In 2002 at the Dr. Lucio Cordova Infectious Diseases Hospital we attended our first confirmed case, a female, HIV (+) patient, C3 CDC stage, with less than $100 \mathrm{CD} 4 / \mathrm{mm}^{3}$ and receiving antiretroviral treatment. She presented a rhinosinusal form and diagnosis was made by CT scan and confirmed with biopsy and culture. This case was treated with amphotericin B deoxycolate and debridement of all necrotic tissue. The patient completed treatment and died one half year after treatment without reactivation of infection.

Key words: Mucormicosis; Rhinosinusal; AIDS.

Palabras claves: Mucormicosis; Rinosinusal; SIDA.

\section{Introducción}

Mucormicosis es una infección rara y oportunista producida por hongos del orden Mucorales, de los cuales las especies que con mayor frecuencia producen enfermedad son Rhizopus, Rhizomucor, Cunninghamella y Absidia. Su distribución es mundial y se encuentran fundamentalmente en la tierra, vegetales en descomposición y el pan. Por su bajo potencial de virulencia raramente producen enfermedad en individuos inmunocompetentes, pero esto cambia radicalmente en pacientes con inmunidad alterada en quienes pueden ocasionar infecciones graves e incluso mortales, de presentación fulminante o lenta e insidiosa. En los pacientes infectados por VIH la mucormicosis es inhabitual; sin embargo, puede ser la infección oportunista actual en SIDA $^{3}$. Describimos a continuación una paciente infectada por VIH que desarrolló una mucor- micosis rinosinusal y fue tratada en el Hospital de Enfermedades Infecciosas Dr. Lucio Córdova.

\section{Caso Clínico}

Mujer de 29 años, portadora de infección por VIH, diagnosticada en 1995, que al momento de su complicación estaba en etapa $\mathrm{C} 3$ de la clasificación CDC, con recuento de CD4 de 96 cél $/ \mathrm{mm}^{3}$ y carga viral de 3.500 .000 copias/ml. Tenía los antecedentes de neumonías a repetición y sinusitis frecuentes, histerectomía por Ca cervicouterino, con recidiva in situ (NIE III) y lúes. En mayo del año 2000 inició terapia anti retroviral (TARV) con zidovudina + lamivudina (Combivir $®)$ y nevirapina, esquema que fue modificado en varias oportunidades por fracaso y mala adherencia. Finalmente se encontraba con TARV de rescate con indinavir, ritonavir, D4T y DDI.

Hospital de Enfermedades Infecciosas Dr. Lucio Córdova:

Servicio de Medicina (LAR, MLQB, MMB, RAV).

Servicio Otorrinolaringología (MRE, MPVU).

Servicio Anatomía Patológica (JCA).

Recibido: 3 septiembre 2003

Aceptado: 24 abril 2004 
Refería además episodios de rinorrea purulenta, obstrucción nasal, anemia y cefalea, con una data mayor a un año.

En julio del 2002 fue rehospitalizada por un cuadro de aproximadamente dos semanas de evolución, caracterizado por odinofagia, tos con expectoración mucopurulenta, fiebre hasta $40^{\circ} \mathrm{C}$ y rinorrea purulenta a lo que se agregó disnea los últimos días. Había recibido en forma ambulatoria penicilina sódica 1 millón UI cada 12 horas durante 4 días, sin notar alivio de sus molestias, por lo que se decidió su internación. Al ingreso se constató fiebre de $38^{\circ} \mathrm{C}$, P. arterial 100/60 mm $\mathrm{Hg}$, pulso $114 / \mathrm{min}$ y polipnea leve. Al examen pulmonar se describió signología bronquial húmeda y sibilancias en ambos campos pulmonares. Los exámenes de laboratorio mostraban $\mathrm{pH} 7,40$, $\mathrm{pO}_{2} 86 \mathrm{mmHg}$, saturación $96,7 \%$, glicemia 82 $\mathrm{mg} / \mathrm{dl}$, nitrógeno ureico $7,0 \mathrm{mg} / \mathrm{dl}$, creatininemia $0,94 \mathrm{mg} / \mathrm{dl}$, hematocrito $29 \%$, recuento de leucocitos $5.800 / \mathrm{mm}^{3}$ y VHS $130 \mathrm{~mm} / \mathrm{h}$. Se plan- teó el diagnóstico de neumonía indicándosele ceftriaxona 1 gr cada 12 horas iv más cotrimoxazol oral $800 \mathrm{mg}$ cada 8 horas. La fiebre se extinguió y la expectoración se aclaró, apareciendo sin embargo, cefalea frontal intensa y compromiso doloroso maxilar bilateral, mayor a derecha. En una fibrobroncoscopia se tomó muestras para búsqueda de virus respiratorios y Pneumocystis jiroveci por IFD, baciloscopia y tinción para Nocardia sp, las que fueron informadas como negativas; además se realizó examen directo y cultivo de hongos. Evaluada por Otorrinólogo se detectó una perforación septal moderada, bordes con costras hemáticas negruscas y una mucosa sangrante. Existía además una candidiasis oral, no se observó rinorrea, masas palpables ni descarga posterior. Se efectuó estudio microbiológico de la mucosa y de las costras. Habiéndose planteado el diagnóstico de mucormicosis se solicitó estudio imagenológico (Figuras 1 y 2). Al día siguiente se informaron los cultivos de hon-
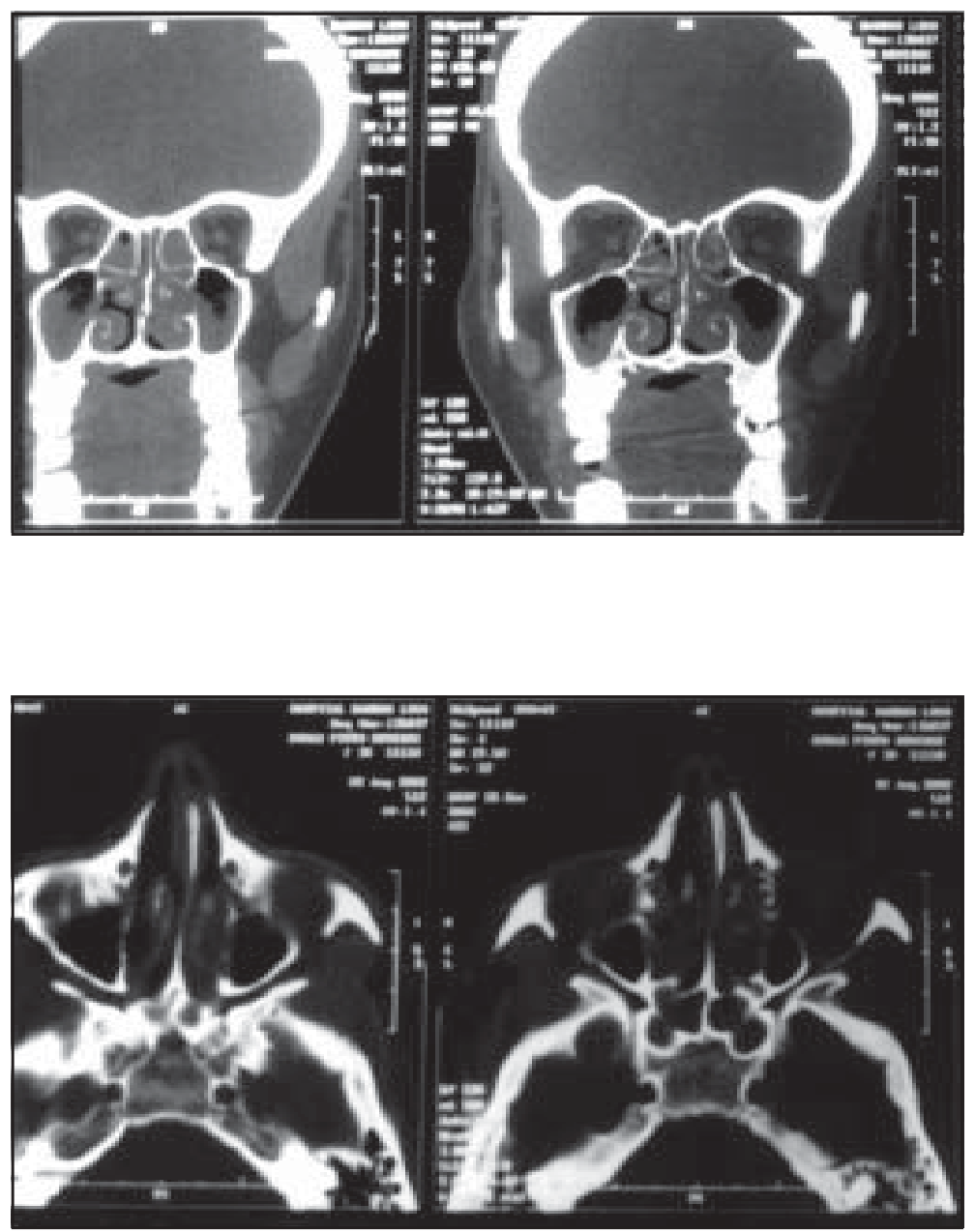

Figura 1. Tomografía axial computada de cavidades paranasales, corte coronal. Se observa velamiento de senos maxilares y etmoidales compatible con sinusitis.
Figura 2. Tomografía axial computada de cavidades paranasales. Se observa ruptura del tabique y compromiso de senos etmoidal y esfenoidal. 


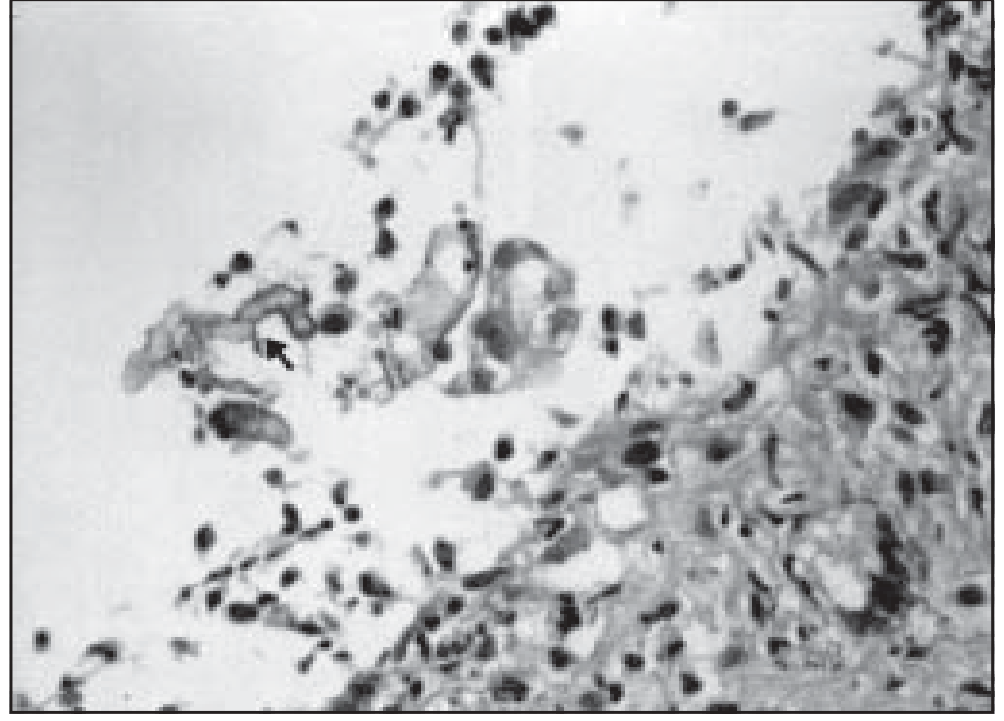

Figura 3. Corte histológico de mucosa nasal que demuestra la existencia de hifas anchas, no septadas, cuya morfología sugiere la presencia de Mucor. gos (LBA y biopsia del septum nasal) como positivos para Mucor spp (Figura 3), por lo que se inició tratamiento con anfotericina $\mathrm{B}$ deoxicolato en dosis de $0,8 \mathrm{mg} / \mathrm{kg} / \mathrm{día}$, y manteniendo terapia antibacteriana. Evolucionó con hiperestesia cutánea en la zona maxilar izquierda y dolor en el ángulo interno del ojo derecho. En la TAC de cavidades paranasales se objetivó una pansinusitis bilateral y una probable lesión ósea del piso orbitario derecho y fosa pterigomaxilar. No se encontró compromiso intracraneano, por lo que se catalogó como mucormicosis rinosinusal no complicada decidiéndose realizar extirpación quirúrgica amplia de toda la zona comprometida: resección del tabique, maxilectomía bilateral, etmoidectomía y esfenoidectomía bilateral. Al sexto día postoperatorio se retiraron los tapones nasales observando un lecho operatorio limpio, sin evidencias de necrosis. Esta zona fue aseada reiteradamente con solución salina fisiológica estéril repitiéndose los aseos quirúrgicos cuando se consideró necesario; en su evolución se observó la zona operatoria limpia, sin costras ni necrosis y sin sangramiento activo, con disminución del edema facial, nariz semipermeable, tos con expectoración purulenta, cefalea frontal, fiebre y calofríos ocasionales, sin compromiso de conciencia. Completó 21 días con ceftriaxona y cotrimoxazol continuando sólo con anfotericina $\mathrm{B}$, ante lo cual se agregó tendencia a la hipokalemia a pesar de los aportes de potasio. Posteriormente se detectó cambios en su personalidad, con rasgos infantiles, sin alteraciones al examen neurológico, descartándose compromiso del lóbulo frontal mediante nueva TAC cerebral. Lue- go recibió nuevamente ceftriaxona + cloxacilina por reaparecer fiebre y rinitis purulenta. En este período la paciente comenzó a presentar náuseas y vómitos en relación con la administración de anfotericina $\mathrm{B}$, se mantenía con hipokalemia a pesar de los aportes de potasio parenteral y además, estaba deprimida. Solicitó el alta cuando completaba $1.875 \mathrm{mg}$ de anfotericina B. Siete días más tarde se volvió a hospitalizar para completar el tratamiento antifúngico, alcanzándose una dosis total de $2.225 \mathrm{mg}$. En un control de TAC se observó una extensa cavidad única nasomaxilar bilateral con evidencias de engrosamiento de la mucosa, cavidades residuales sin signo de lesión ósea y sin compromiso del parénquima cerebral. Con posterioridad al alta se hospitalizó en varias oportunidades por neumonías, en ocasiones extensas y graves, o síndrome febril sin etiología clara, con buena respuesta a los tratamientos antibacterianos sin demostrarse reactivación de la mucormicosis. Estando sin TARV por una mala respuesta (clínica, inmunológica y virológica), experimentó un deterioro progresivo de su estado general, anemia persistente, y falleció en diciembre del año 2003.

\section{Comentario}

La mucormicosis es muy inhabitual en los enfermos infectados por VIH; sin embargo, se han descrito casos desde $1989^{1}$, con localizaciones rinocerebral, pulmonar, cutáneo-articular, renal y diseminada ${ }^{2-5}$. Ocurre primariamente en pacientes con muy bajo recuento de linfocitos 
CD4, generalmente $<100$ céls $/ \mathrm{mm}^{3}$ (con un rango de 0 a 387 céls $\left./ \mathrm{mm}^{3} \mathrm{l}\right)$. Otros factores predisponentes en estos pacientes son: neutropenia, drogadicción iv (que se caracteriza por compromiso de ganglios basales) y diabetes mellitus.

La forma rinosinusal que presentó nuestra paciente generalmente se asocia con diabetes mellitus; se ha observado que aproximadamente dos tercios de estos pacientes son diabéticos ${ }^{6}$; la misma relación se encuentra en los pacientes infectados por VIH, pero no ocurrió así en el caso que presentamos.

No son muchos los casos de mucormicosis publicados en nuestro país, en adultos o en niños ${ }^{7-10}$. En ellos se ha visto asociada a diabetes mellitus e inmunosupresión por enfermedades hematológicas o neutropenias producidas por fármacos. No se ha comunicado antes en Chile mucormicosis en pacientes infectados por VIH.

Nuestra paciente tenía más de siete años de evolución de su infección por VIH (fue diagnosticada en el año 1995), con recuentos de CD4 de 96 céls $/ \mathrm{mm}^{3}$, episodios de neutropenia frecuentes y desnutrición, mala adherencia a los TARV, habiendo recibido varios esquemas distintos por fracaso o mala tolerancia. En su evolución presentó múltiples episodios de infecciones respiratorias, sinusitis, neumonías, las que fueron motivo de hospitalizaciones reiteradas. No tenía antecedentes de drogadicción iv o diabetes mellitus.

En la forma rinocerebral, las manifestaciones clínicas dependerán de la velocidad de progresión de la infección y de las estructuras intracerebrales comprometidas. La infección nasal sigue a la inhalación de aerosoles de esporas del hongo, éstas se depositan en los cornetes nasales y se extienden a los senos paranasales y órbitas. Este patógeno invade y se disemina a través de los vasos sanguíneos, particularmente las arterias, el hongo prolifera dentro de la lámina elástica interna y diseca hacia la media. Una vez que las hifas penetran el endotelio se producen arteritis trombóticas, infartos, hemorragias y extensa necrosis tisular ${ }^{6,11-14}$. La infección con Aspergillus sp, Pseudomonas aeruginosa o Staphylococcus aureus también propende a la invasión vascular con producción de infartos tisulares, por lo que deben ser contemplados en el diagnóstico diferencial $^{11-13}$, al igual que la celulitis orbitaria bacteriana, o la trombosis del seno cavernoso.

Los pacientes con mucormicosis rinocerebral generalmente presentan letargia, fiebre, y cefalea o dolor facial. Aparece una escara necrótica, negra, intranasal o en el paladar, aspecto altamente sugerente de la enfermedad pero que sólo está presente en $40 \%$ de los pacientes. La extensión de la enfermedad hacia la órbita o los senos cavernosos determina la producción de celulitis periorbitaria, parálisis de los músculos extraoculares, proptosis y quemosis. Luego pueden presentar compromiso de pares craneanos, pérdida de la visión por oclusión de la arteria central de la retina, extensión directa a la órbita y finalmente, signología neurológica que estaría determinada por la formación de abscesos cerebrales $^{6,11,13-16}$.

Nuestra paciente presentó un cuadro caracterizado por cefalea, hiperestesia cutánea, dolor facial y fiebre. No tuvo compromiso de conciencia, alteración de pares craneanos, ni signos de celulitis orbitaria con pérdida de la visión; tampoco presentó alteraciones inflamatorias de los tejidos blandos en la zona afectada. Sólo tenía el antecedente de descarga nasal de tipo purulento. En ella el examen otorrinológico fue determinante para establecer una sospecha diagnóstica, la que fue confirmada por biopsia y cultivo. La TAC reveló el grado de extensión de la lesión y demostró que no había compromiso cerebral pero sí existían alteraciones sugerentes de compromiso del piso de la órbita, lo que fue descartado durante la cirugía.

El diagnóstico de mucormicosis se basa en tres elementos fundamentales: la sospecha clínica (existencia de factores de riesgo, cuadro clínico sugerente), los estudios e imágenes, y los exámenes microbiológicos e histológicos.

Las radiografías de senos paranasales y de órbitas pueden mostrar algunas alteraciones, como engrosamiento de la mucosa sinusal, con o sin niveles hidroaéreos. Si la enfermedad está más avanzada, se puede observar erosión del hueso de las paredes de los senos o en la órbita. Todo esto es puesto de manifiesto con mayor detalle y en toda su extensión por la TAC, la que indica hasta dónde debiera extenderse la resección quirúrgica. La RM muestra cambios similares, pero con mayor detalle $e^{6,11,13,14}$.

El diagnóstico etiológico comprende el examen microscópico directo, que mostrará la presencia de hifas gruesas, como cinta, de 10-20 $\mu$, no septadas, con ramificaciones en ángulo recto. El cultivo permite la identificación del género y la especie, lo que sirve de orientación para el tratamiento antimicótico; sin embargo, un cultivo positivo no tiene valor diagnóstico, ya que podría corresponder a una simple colonización ${ }^{6,11,14,17}$. Una técnica rápida que se ha demostrado de utilidad para el diagnóstico de esta patología, es la tinción de blanco de calcoflúor, la que permite visualizar las estructuras fúngicas claramente $\mathrm{y}$ 
se puede poner en práctica en biopsias rápidas durante el acto operatorio ${ }^{8,17}$. Los tests de inmunodiagnóstico (inmunodifusión y EIA) han resultado poco sensibles e inespecíficos. La RPC puede llegar a ser una modalidad diagnóstica para identificar en forma precoz estos organismos, pero aún no está disponible para su uso en clíni$\mathrm{ca}^{15-17}$. El diagnóstico confirmatorio es el examen histopatológico, considerado el patrón de oro con estos fines. Con esta técnica se demuestra la invasión de la pared vascular y del tejido por las hifas no septadas, en ángulo recto y áreas de necrosis e infartos con vasculitis, trombosis y hemorragias que serían lo característico de esta enfermedad ${ }^{6,11,13,14,16}$.

Los pilares fundamentales del tratamiento son la terapia antifúngica con alguna preparación de anfotericina $\mathrm{B}$, cirugía extensa y radical y en lo posible, la corrección de las condiciones predisponentes (por ej cetoacidosis diabética, inmunosupresión etc) $)^{6,7,11,14}$.

La anfotericina $\mathrm{B}$ es el antifúngico de elección para el tratamiento de la mucormicosis. Debido a que el hongo presenta una resistencia relativa, tanto clínica como microbiológica al fármaco, se recomiendan dosis más altas que las habituales, es decir, 1 a 1,5 mg/kg/día. La duración de la terapia no está claramente definida. En muchos estudios la dosis alcanzada empleada ha sido de al menos 2 gramos y algunos pacientes han recibido más de 4 gramos ${ }^{6,9,11,13,14}$.

Las formulaciones lipídicas de anfotericina B son una alternativa apropiada a la anfotericina B convencional y pueden representar un gran avance en estos tratamientos. Clínicamente son tan eficaces como la anfotericina convencional, pero mucho mejor toleradas debido a que son significativamente menos nefrotóxicas y producen muy poca reacción durante la infusión iv. Esto mismo permite usar dosis más altas que la anfotericina convencional, pudiendo llegar a emplearse $10 \mathrm{mg} / \mathrm{kg} /$ día, lo que podría determinar una mejor respuesta al tratamiento y mayor sobrevida. Limita bastante su uso el elevado costo ${ }^{6,19,20}$.

Otra forma de reducir la toxicidad de anfotericina convencional podría ser el uso en infusión continua, pero esto significa administración permanente y la posibilidad de interacciones con otros fármacos ${ }^{21}$.

Una alternativa válida y que reduce significativamente la nefrotoxicidad es el suplemento de potasio, sodio y magnesio correspondientes a las cantidades perdidas por el riñón, junto a la hidratación rigurosa (mantener una diuresis diaria de más o menos 4 litros) $)^{22}$.
De los nuevos azoles, voriconazol no es activo contra los agentes de mucormicosis al igual que ravuconazol. Posaconazol tiene mejor actividad in vitro que los otros azoles y se ha empleado con éxito, por lo menos, en un caso $^{23}$. Las equinocandinas como caspofungina, anidulafungina y micafungina, también son inactivas, con algunas excepciones, contra Zigomycetes ${ }^{16,18,24}$.

El oxígeno hiperbárico también ha sido de valor en el tratamiento de la mucormicosis. Varios estudios han publicado el uso exitoso de oxígeno hiperbárico en mucormicosis rinocerebral, cutánea y de tejidos blandos, como terapia asociada a la cirugía y anfotericina $\mathrm{B}$, pero en algunas publicaciones se han encontrado sesgos que hacen difícil interpretar estos datos con seguridad $^{6,15}$.

Aunque han aparecido en la literatura médica reportes de recuperación con tratamiento antimicótico solo, éstas son la excepción ${ }^{4}$, por lo que se recomienda una extirpación extensa y amplia de todo el tejido necrótico hasta que los márgenes se vean libres de infección, para impedir que el hongo continúe multiplicándose e invadiendo tejido sano. En ocasiones se requiere más de un aseo quirúrgico; si es posible, debe ser guiado por técnicas de biopsia rápida para que la cirugía no sea tan mutilante ${ }^{7,11,14}$.

Nuestra paciente fue tratada con anfotericina B alcanzando una dosis total de $2.225 \mathrm{mg}$, más extirpación de todo el tejido necrótico, cirugía que se repitió en más de una oportunidad.

La mucormicosis rinocerebral era considerada mortal, sin tratamiento la infección llevaba rápidamente al desenlace fatal. Sin embargo, en los últimos veinte años ha mejorado el pronóstico. Se han publicado tasas de sobrevida superiores a $85 \%$ y para ello es fundamental el diagnóstico precoz, la corrección de las condiciones predisponentes, tratamiento antifúngico adecuado e intervención quirúrgica agresiva ${ }^{6,11,15}$.

\section{Resumen}

Mucormicosis es una infección fúngica oportunista producida por varias especies del orden Mucorales, como Rhizopus, Mucor, Cunninghamella y Absidia. Habitualmente se asocia con enfermedades oncohematológicas, diabetes mellitus, falla renal, tumores sólidos y trasplante de órganos sólidos o precursores hematopoyéticos. Se presenta con una variedad de formas clínicas: rinocerebral, renal, pulmonar, cutánea, gastrointestinal y diseminada. En pacientes infectados por VIH es poco frecuente. Presentamos una paciente atendida en el Hospital de Enfermedades Infecciosas Dr. Lucio Córdova, con infección por VIH en etapa C3 del CDC, CD4 < 100 céls $/ \mathrm{mm}^{3}$ y 
tratamiento anti retroviral. La forma de presentación rinocerebral fue documentada por TAC que demostró la extensión de la lesión, y confirmada mediante biopsia y cultivo. Recibió anfotericina B más resección quirúrgica de todo el tejido necrótico. Completó $2.225 \mathrm{mg}$ del antifúngico falleciendo un año y medio después, sin que se demostrara reactivación de la infección.

\section{Bibliografía}

1.- Smith A, Bustamante C, Gilmor G. Zygomycosis (absidiomycosis ) in an AIDS patient. Absidiomycosis in AIDS. Mycopathologia 1989; 105: 7-10.

2.- Abril V, Ortega E, Segarra P, Pedro F, Sabater V, Herrera A. Rhinocerebral mucormycosis in a patient with AIDS: A complication of diabetic ketoacidosis following pentamidine therapy. Clin Infect Dis 1996; 23: 845-6.

3.- Moraru R, Grossman M. Palatal necrosis in an AIDS patients. A case of mucormycosis. Cutis 2000; 66 (1): $15-8$.

4.- Weng D, Wilson W, Little R, Walsh T. Successful medical management of isolated renal zygomicosis: Case report and review. Clin Infect Dis 1998; 26: 601-5.

5.- Virally M, Riveline J P, Virally J, Chevojon P, Regnard $\mathrm{J}$ F, Belmekki A, et al. Pulmonary mucormycosis in a diabetic patient with HIV. Diabetes Care 2002; 25 (11): 2105

6.- González C, Rinaldi M, Sugar A. Zygomycosis. Infect Dis Clin North Am 2002; 16: 895-914.

7.- Bravo M, Ferrer S, Etchard M, Trujillo S. Mucormicosis rinocerebral. Comunicación de 4 casos. Rev Méd Chile 1999; 127: 712-8.

8.- García P, Beltrán C, Guzmán A, León P, Arredondo M y Fonseca X. Diagnóstico rápido de dos casos de mucormicosis con tinción de blanco de calcoflúor. Rev Chil Infect 2001; 18 (4): 285-90.

9.- Wicki A, Borel C, Villarroel M. Cofré J. Curación de mucormicosis rinocerebral en un paciente pediátrico con leucemia activa. Rev Chil Infect 2004; 21: 53-6.

10.- Spalloni W Glaser P, Verdugo P. Mucormicosis rinocerebral: Sobrevida en un niño con leucemia. Rev Chil Infect 2004; 21: 56-9.

11.- Sugar A. Agents of mucormycosis and related species. En Mandell, Douglas and Bennett's: Principles and
Practice of Infectious Diseases. Mandell G L, Bennett J E, Dolin R eds. Fifth Edition. Churchill. Livingstone, Philadelphia 2000; 3250-7.

12.- Petrikkos G, Skiada A, Sambatakou H, Toskas A, Vaiopoulos G, Giannopoulou M, et al. Mucormycosis: Ten-year experience at a tertiary-care center in Greece. Eur J Clin Microbiol Infect Dis 2003; 22 (12): 753-6.

13.- Sugar A M. Mucormycosis. Clin Infect Dis 1992; 14 (Suppl 1): S 126-9.

14.- Weprin B, Hall W, Goodman J and Adams G. Longterm survival in rhinocerebral mucormycosis. J Neurosurg 1998; 88: 570-5.

15.- Prabhu R, Patel R. Mucormycosis and entomophthoramycosis: a review of the clinical manifestations, diagnosis and treatment. Clin Microbiol Infect 2004; 10 (Suppl 1): 31-47.

16.- Spalloni W, Chávez A, Avilés C, Cofré J. Mucormicosis en Pediatría. Rev Chil Infect 2004; 21: 17-25.

17.- Guzmán A. Importancia del laboratorio en el diagnóstico de las micosis invasoras. Rev Chil Infect 2004; 21: $39-47$.

18.- Jacobs P, Wood L, Du Toit A, Esterhuizen K. Erradication of invasive mucormycosis. Effectiveness of the echinocandin FK463. Hematology 2003; 8: 119-23.

19.- Jiménez C, Lumbreras C, Aguado J M, Loinaz C, Paseiro G, Andres A, et al. Successful treatment of mucor infection after liver or pancreas-kidney transplantation. Transplantation 2002; 73 (3): 476-80.

20.- Moses A E, Rahav G, Barenholz Y, Elidan J, Azaz B, Gillis S, et al. Rhinocerebral mucormycosis treated with amphotericin B colloidal dispersion in three patients. Clin Infect Dis 1998; 26 (6): 1430-3.

21.- Inhof A, Walter R, Schaffnes A. Continuous infusion of escalated doses of amphotericin B deoxycholate: An open-label observational study. Clin Infect Dis 2003; 36: 943-51.

22.- Mayer J, Doubek M, Doubek J, Horky D, Scheer P, Stepanek M. Reduced nephrotoxicity of conventional amphotericin B therapy after minimal nephroprotective measures: Animal experiments and clinical study. J Infect Dis 2002; 186 (3): 379-88.

23.- Tobar A, Arango M, Fernández D, Restrejo A. Mucormycosis (zygomycosis) in a heart-kidney transplant recipient: Recovery after posaconazole therapy. Clin Infect Dis 2003; 36: 1488-91.

24.- Groll A H, Gea-Banacloche J C, Glasmacher A, JustNuebling G, Maschmeyer G, Walsh T J. Clinical pharmacology of antifungal compounds. Infect Dis Clin North Am 2003; 17 (1): 159-91.

Correspondencia a:

Lucía Aguad Readi

luciosubdirmed@ssms.cl 